\title{
Thermal tomography on the basis of an information method
}

\author{
By S. Melnyk*
}

${ }^{*}$ Kharkov National University of Radio- Electronics, Kharkov, Ukraine

\begin{abstract}
A unified (information) approach to the development of an optimum algorithm of thermal tomography on the basis of the available prior information has been designed. The essence of the new criterion is the minimization of the complexity of the description of experimental results. It increases a resolution of thermal monitoring by several folds. The problem of the testing of delamination in a multilayer object is considered as an example of usage. The capability of detection and determination of delamination borders is shown; if its size is much smaller than the depth and thermal resistance is small.
\end{abstract}

\section{Introduction}

The development of thermovision engineering allows one to solve the problem of thermal tomography, in addition to thermal non-destructive testing and defectometry problems. Currently, the basic method employed in practice is the pulse thermal tomography. As a matter of fact, the one-dimensional model of a heat flux is used in it. Only one parameter - the depth of location of a not heat-conducting imperfection is determined. Thus only a small part of the information about interior structure of the monitored object contained in thermovision film is used. The purpose of this work is the development of a new universal method of solving the thermal tomography problem. It is based on full usage of both the thermovision information and prior information on the object of monitoring. Mathematical techniques of algebraic information theory ("the theory of complexity") [3] allow for solving this problem.

\section{The essence of the method}

The problem of the testing of delamination in a multilayer object is considered as an example of usage. Any local non-uniformity of thermophysical properties of the object of monitoring (imperfection) can be represented by an equivalent heat source and heat dipole, distributed accordingly in a volume and on a surface of this nonuniformity [1]. The power of an equivalent heat dipole is proportional to the thickness of an air interlayer and power of a heat flux. Thus, the thermal tomography of separations is reduced to determination of a map of thermal dipoles located on one of given boundaries of layers. In the elementary stationary case of two-dimensional model, the temperature response to the surface of the object can be calculated as follows:

$$
T(x)=\sum_{i=1}^{k} G_{i}(x) * q_{i}(x)+\delta T_{s}(x),
$$


where $G_{i}(x)$ is the thermal transfer function of $i$ layers placed above the assigned equivalent thermal dipole of power $q_{i}(x) ; \delta T_{s}(x)$ is additive noise with a known spectrum; * stands for convolution. Thus the problem of the determination of $q_{i}(x)$ from known $T(x)$ and $G_{i}(x)$ is ill-posed.

A major problem that appears in the process of the solution of the abovementioned inverse task is a choice between regularization criteria. With this purpose, many known methodologies use a priori information about a signal and a noise component. As a rule, this component is the noise amplitude. In the case of a stochastic signal, this can be information about the spectrum of the signal and noise (i.e., optimum spectral filtration method).

We can state that in these and other cases, they actually minimize complexity of description of experimental results, which are set up at a definite accuracy. Hereby, a priori information is required at choice for description- algorithm. Hence, in particular, follows a minimization criterion for root-mean-square deviation for the case of normal distribution of the noise component, Shannon's formula for random signal entropy evaluation and many others.

For this reason, the principle of minimized complexity of the description of experimental results can be used as a universal criterion for optimization of the solution of the inverse task. (By the way, this method is an integrated principle of entropy maximum). Hereby, a priori information on signals or noises can be considered as additional preliminarily obtained experimental results.

If a tested object is assumed to be a multilayer plate with delamination, such information can include profile (probably, rectangular, Gausse-tipe) of delamination, depth of location for every individual layer, etc.

The practical accessibility of different estimations of a thermal monitoring resolution depends on the available prior information. So, for example, usage of the information that the defects are small local patches on given depth (can be described by $\delta$ - functions), allows to increase a resolution of thermal monitoring by several fold. The results of the computer experiments with two small defects are shown in figure 1.

\section{The pulse thermal tomography}

The essence of the pulse thermal tomography is the harmonic heat action at the object surface and the measurement of the phase lag of its temperature. The results of the testing are the thickness of the plate or the depth of the delamination. Let's note that the method of pulse thermal tomography solves the problem of the delamination defectometry when the sizes of local separations exceed their depth, and the thermal resistance of separation exceeds thermal resistances of above located layers.

The solution of the heat transfer equation for the uniform plate by the heat transfer function method gives a constraint on the space time spectrum of the temperatures $T_{1}(x, y, \tau), T_{2}(x, y, \tau)$ and heat fluxes $q_{1}(x, y, \tau), q_{2}(x, y, \tau)$ at the both surfaces of the plate [2]

$$
\left|\begin{array}{l}
\widetilde{T}_{2}\left(w_{x}, w_{y}, w\right) \\
\frac{1}{k \lambda} \widetilde{q}_{2}\left(w_{x}, w_{y}, w\right)
\end{array}\right|=\left[\begin{array}{ll}
\cos (k h) & -\sin (k h) \\
\sin (k h) & \cos (k h)
\end{array}\right] \cdot\left|\begin{array}{l}
\widetilde{T}_{1}\left(w_{x}, w_{y}, w\right) \\
\frac{1}{k \lambda} q_{1}\left(w_{x}, w_{y}, w\right)
\end{array}\right|,
$$


where $\widetilde{T}_{1}, \widetilde{T}_{2}, \widetilde{q}_{1}, \widetilde{q}_{2} \quad$ are obtained with Fourier transform; $k^{2}=-i \cdot w / a-w_{x}^{2}-w_{y}^{2} ; a=\lambda /(c \rho)$. We can see that the ratio of the heat flux and temperature spectrum at the different depths is connected with Eq. (3).

$$
\operatorname{tg} \xi_{2}=\operatorname{tg}\left(\xi_{1}+k h\right), \text { where } \operatorname{tg} \xi_{i}=\frac{\widetilde{q}_{i}\left(w_{x}, w_{y}\right)}{k \lambda \widetilde{T}_{i}\left(w_{x}, w_{y}\right)} ; i=1,2
$$

For the one-dimension model and $q_{2} \equiv 0$ (non-heat-conducting delamination) Eq. (3) transforms to the phase lag equation

$$
\delta \varphi\left(\frac{T_{1}}{q_{1}}\right)=\delta \varphi[k \lambda \cdot \operatorname{tg}(k h)], \quad \text { where } \quad k^{2}=-i \frac{w}{a} .
$$

That's just what is used in the majority methodic of the pulse thermal tomography for the $h(x, y)$ determination. We can allow for the heat exchange at the surfaces of the plate by the following equation:

$$
\delta \varphi\left(\frac{T_{1}}{Q_{1}}\right)=\delta \varphi\left(\frac{k \lambda-\alpha_{2} \operatorname{tg}(k h)}{k \lambda\left(\alpha_{1}+\alpha_{2}\right)-\left(k^{2} \lambda^{2}+\alpha_{1} \alpha_{2}\right) \operatorname{tg}(k h)}\right),
$$

where $\alpha_{1}$ and $\alpha_{2}$ are the effective coefficients of the heat transfer at the heated surface and the surface of the defect, respectively.

We can't use the one-dimension approximation in the general case. For example, let us consider a thin delamination with the varying thickness $d(x, y)$,

$$
\delta T_{d}(x, y)=q_{2}(x, y) \cdot d(x, y) / \lambda_{d}(x, y)=q_{2}(x, y) \cdot R(x, y),
$$

where $\delta T_{d}(x, y)$ is the temperature drop at the delamination; with $R(x, y)$ being its heat resistance. The region behind the delamination is assumed to be homogeneous and thick enough. Then the spectrum of the temperature as a function of the heat flux spectrum at the boundary of this region is

$$
\widetilde{T}_{2}\left(w_{x}, w_{y}\right)-\delta T_{d}\left(w_{x}, w_{y}\right)=\widetilde{q}_{2}\left(w_{x}, w_{y}\right) /\left(i k \lambda_{d}\right)
$$

From Eqs. (2), (6) and (7) we obtain

$$
R(x, y)=\frac{\delta T_{1}(x, y) * F^{-1}[\exp (i k h)]}{\delta q_{2}(x, y)+q_{1}(x, y) * F^{-1}[\exp (-i k h)]},
$$


where $\delta q_{2}(x, y)=\delta T_{1}(x, y) * F^{-1}[k \lambda \cdot \sin (k h)]$ is the heat dipole which is equivalent to the delamination; $\delta T_{1}(x, y)=T_{1}(x, y)-T_{n d}(x, y)$ is a complex valued temperature drop at the surface of the tested object, which is caused by delamination, $T_{n d}(x, y)$ is the temperature at the surface of the non-defect object.

The tasks of the $\delta T_{d}(x, y)$ and $\delta q_{2}(x, y)$ determination are the inverse illposed problems. One of the regularization methods is used for them. The information on the phase lag $\delta \varphi(x, y)$ alone is not enough in the two-dimensional problem. That is why the nonuniformity of the surface is the main factor of the sensitivity of the thermal testing method. In this case the inverse task transforms to

$$
\begin{aligned}
& T_{1}(x, y) \cdot\left[\varepsilon_{0} / \varepsilon(x, y)\right]-T_{n d}=\left(F^{-1}[\exp (i k h)]\right)^{-1} * \delta T_{d}(x, y), \\
& \text { or as in Eq. (1) } \delta T_{1}(x, y)=G_{h}(x, y) * \delta T_{d}(x, y)-T_{1}(x, y) \cdot \frac{\delta \varepsilon}{\varepsilon}
\end{aligned}
$$

For the stationary thermal testing method $(w=0)$ we have the familiar result $G_{h}\left(w_{x}, w_{y}\right)=\exp \left[-h \sqrt{w_{x}^{2}+w_{y}^{2}}\right\rfloor$. The high frequencies of the signal decay exponentially with the distance and the information on the delamination shape is lost.

\section{A priory information as the part of the reconstruction method}

The new criterion is the "minimization of algorithmic complexity of description of experimental data including all available prior information". Thus prior information can be formalized and represented by a set of coding algorithms. They are used as the components at build-up of a procedure of the inverse problem solution.

At first, let us consider the main optimization criterion for the ill-posed problem. With regard to $q_{i}\left(w_{x}\right)$, solution of (1) takes the form

$$
q_{i}\left(w_{x}\right)=\left(\frac{1}{1+\alpha\left(w_{x}\right)}\right) \cdot \frac{G^{*}\left(w_{x}\right) \cdot T\left(w_{x}\right)}{G^{*}\left(w_{x}\right) \cdot G\left(w_{x}\right)}, \quad \alpha\left(w_{x}\right)=\frac{\delta T_{s}\left(w_{x}\right)}{G\left(w_{x}\right) \cdot q_{i}\left(w_{x}\right)}
$$

Now, we can see that parameter $\alpha\left(w_{x}\right)$ is responsive to influence of noises, because in their absence, it will turn to zero. However, we cannot calculate this parameter precisely unless we obtain the task solution. In the evaluation of the solution according to the above formula, required a priori information is used. The expression $\left[1+\alpha\left(w_{x}\right)\right]^{-1}$ is known as the stabilizing multiplier. Its purpose is to neutralize an effect of solution- instability, owing to growth of multiplier at elevation of frequency. Tikhonov has shown that for convolution- type equation, a set of stabilizing multipliers of common form, such as: $\alpha(w)=\alpha_{0} \sum_{n=0}^{p} c_{n} w^{2 n}$ leads to minimization of functional equation: 


$$
M^{\alpha}(q, T)=\int_{-\infty}^{\infty}(G * q-T)^{2} d x+\int_{-\infty}^{\infty} \alpha(w)|q(w)|^{2} d w
$$

where the former component is proportional to R.M.S. deviation and the latter is to compensate for large values of amplitude and derivatives from the obtained solution. In this manner, choice of algorithm for regularization of non- correct task- solution is based on a priori- information on smoothness and limits of desirable solution. Thus this algorithm of regularization can be represented as a particular case of the new universal criterion.

We can take account not only of the spectrum properties of the signal and the noise but of any prior information on them also with the new criterion. We calculate the minimized functional equation as

$$
M_{\text {comp }}(q, T)=\operatorname{Comp}[T]=\operatorname{Comp}\left[q_{h}, \delta q_{h}\right]+\operatorname{Comp}\left[G * q-T, \delta q_{h}, \delta T\right]
$$

The function of the complexity calculation "Comp" is varied with the kind of the priory information. The accuracy of $q(x, y)$ description is the varied parameters of the function "Comp", but the accuracy of $T(x, y)$ description is an input parameter. The requirement of uncancellability of description complexity of input data for a direct problem is available to a minimum of information criterion (in practice this requirement is equivalent to lack of unaccounted regularities in description of real monitored object properties).

The procedure of the solution of a thermal tomography problem by information method includes the following points:

- To formalize the solution of a direct problem - mathematical model of non destructive testing process (the previous part of this report for example)

- To develop the algorithms of optimum algorithmic description ("Comp" functions) of all data necessary for the solution of a direct problem (as required parameters of object of monitoring, and parameters of the defects, and noise);

- To develop the minimization algorithm of complexity of the thermal monitoring data;

- To develop the algorithm of the thermal testing parameters optimization.

\section{Results of the numerical simulation}

The capability of the detection and determination of delamination borders and width as functions of coordinates is illustrated in figure 2 (compared with other methods).

\section{References}

1 Storozhenko V.A., Melnik S.I., Orel R.P. The New Algorithms of Thermal Defectometry. // 10th International THERMO Conference, 18-20 June, 1997, Budapest, Hungary.

2. Storozhenko V.A., Melnik S.I. Development of the Thermal Defectometry on the Base of the Transfer Function Method //Abstracts of the 8th International Conference THERMO-93,Hungary, Budapest, 1993.

3. Kolmogoroff A.N. .Logical basis for information theory and probability theory.-IEEE Trans. Inform. Theory, 1968, vol. IT-14, p. 662-664. 


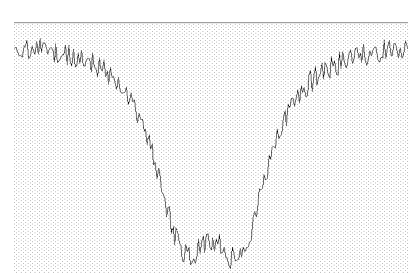

a)

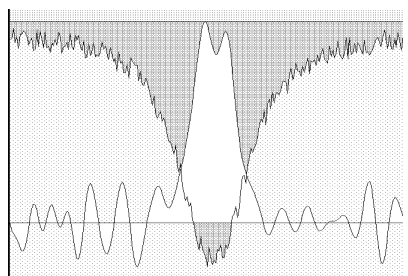

b)

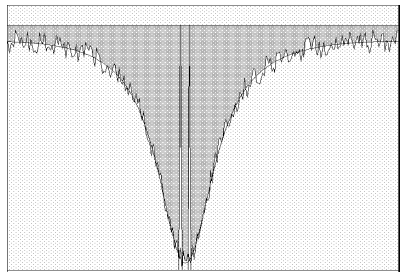

C)

Fig. 1 The resolution of thermal nondestructive testing

a) the raw registered signal makes 1.7 of the depth of local defects

b) the result of the inverse problem solution by Tihonov regularization method makes 0.6 of the depth of local defects

c) the solution obtained in view of the information on the shape of the defects makes 0.2 of the depth of local defects

(a noise level is equal to $5 \%$ ).

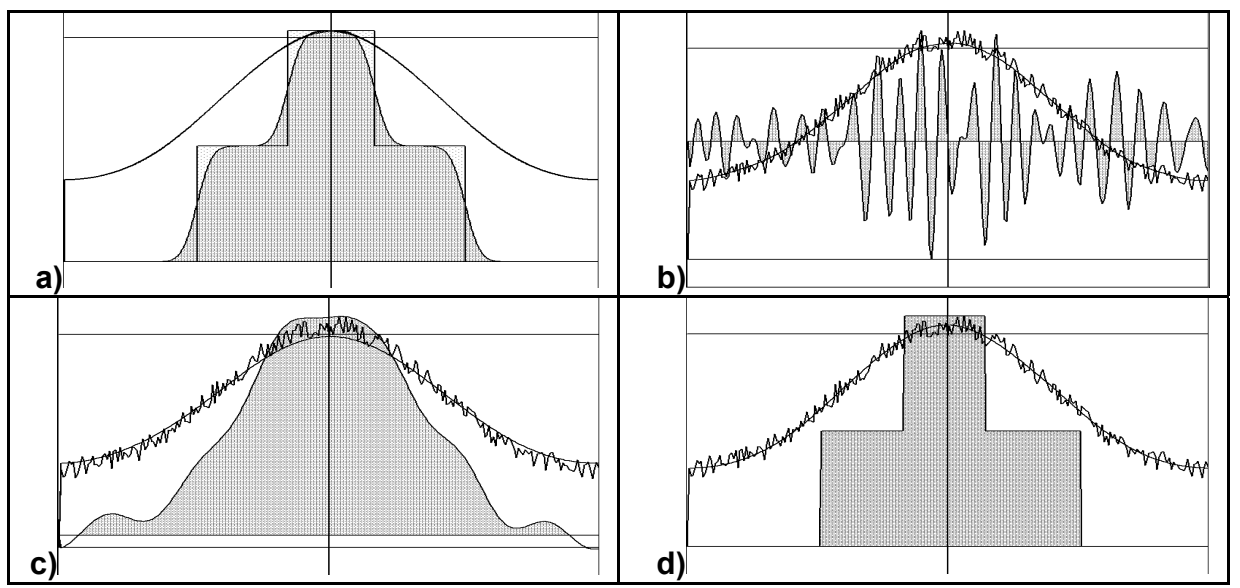

Fig. 2 The restoration of the shape of the delaminating

The width of the delaminating is equal to its depth.

a) Noise level is equal 0 . The results of the restoration by Tihonov regularization and "Comp" methods.

b) Noise level is equal $5 \%$. The result of the restoration by R.M.S. minimization method.

c) Noise level is equal $5 \%$. The result of the restoration by Tihonov regularization method.

d) Noise level is equal $5 \%$. The result of the restoration by "Comp" method. 